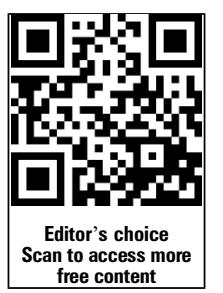

${ }^{1}$ Rehabilitation Medicine and Rheumatology, Defence Medical Rehabilitation Centre, Epsom, UK

${ }^{2}$ Neuro-rehabilitation Medicine, Defence Medical Rehabilitation Centre, Epsom, UK

${ }^{3}$ Defence Rehabilitation, Defence Medical Rehabilitation Centre, Epsom, UK

\section{Correspondence to}

Major Sardar Bahadur, Rehabilitation Medicine and Rheumatology, DMRC, Epsom, UK:

sbahadur@nhs.net

Received 2 December 2014 Revised 18 August 2015 Accepted 20 August 2015 Published Online First 18 September 2015

\title{
Injury severity at presentation is not associated with long-term vocational outcome in British Military brain injury
}

\author{
Sardar Bahadur, ${ }^{1}$ E McGilloway, ${ }_{1}$ J Etherington ${ }^{3}$
}

\section{ABSTRACT}

Introduction Injury Severity Score (ISS) and GCS can be retrospective markers of injury severity, but if used by clinicians to decide on the treatment of acutely braininjured casualties at the point of injury may potentially limit interventions on people who may ultimately survive with good functional outcomes.

Methods ISS/GCS and long-term outcomes were reviewed by assessing all UK military neurorehabilitation patients with an operational/combat brain injury treated over 4 years (February 2008-July 2012) at Defence Medical Rehabilitation Centre (Headley Court).

Results 34 participants from 9 operational tours of Iraq and Afghanistan were analysed. Overall, $44 \%$ of injuries were due to improvised explosive devices (IEDs) and $41 \%$ from gunshot wounds; $70.9 \%$ of injuries were penetrating wounds with the remainder due to blast/ blunt trauma or combined injury. The primary injury was head/neck in $76.5 \%$, although eight patients (23.4\%) requiring neurorehabilitation were initially 'non-head injury'. Eight patients (26.5\%) sustained more than 10 injuries, and 18 had between three and nine injuries. Eleven patients (32\%) had an initial GCS of 3 , and $16(47 \%)$ had ISS of 75 (deemed 'unsurvivable'). All patients with ISS of 75 were long-term survivors. At 4 months after discharge, 47\% (16) were fully independent, and a further $41 \%$ (14) were independent in own homes, but needed assistance with some activities, such as paying bills. Over three-quarters (27 patients, $79 \%$ ) returned to full/part-time work, 11 of whom returned to military duties; $93 \%$ of 'unsurvivable' ISS, and $91 \%$ of patients with GCS of 3 were capable of returning/returned to work. In total, 7/11 casualties returning to military duties had major trauma ISS, and two were 'unsurvivable'. All seven casualties with both GCS 3 and ISS 75 survived and returned to independence (help with some activities).

Conclusions ISS/GCS at the point of injury does not reflect eventual outcome. IEDs/gunshots cause the greatest number of injuries and the highest incidence of brain injury. Brain injury should be considered in every battlefield casualty, irrespective of whether the head/neck/ spinal cord was avoided. ISS should not be considered indicative or predictive of long-term prognosis/quality of life/employability as brain injury in this small cohort is both survivable and recoverable. It should not be used as a retrospective guide to alter treatment pathways, as there is poor correlation with long-term outcome. Subsequent neurorehabilitation should always be considered because survival, return to independence and full employment are very likely.

\section{Key messages}

Brain injury should be considered in every casualty involved in battle, irrespective of whether the primary injury avoided the head/ neck/spinal cord.

- Injury Severity Scores should not be considered indicative or predictive of long-term prognosis, quality of life or employability as brain-injury is both survivable and recoverable.

- Most brain injury in military personnel is caused by IED attacks and Gunshot wounds.

- Neuro-rehabilitation should always be considered as not only survival but return to independence and full employment is very likely.

- Frontline military clinicians should be encouraged to actively intervene and treat patients with severe traumatic brain injury in the knowledge that full recovery is possible.

\section{INTRODUCTION}

Injury assessment in military and civilian settings forms the cornerstone of initial life-sustaining intervention, subsequent life-prolonging management and future planning to improve long-term outcomes. Urban, rural and battlefield environments seemingly represent completely different conditions for casualty management, yet the ambition is the same for all-to retrieve the casualty to a place of medical expertise where all injuries can be assessed and treatment planned. Initial assessments are encouraged to be comprehensive, aiming to detect all injuries and identifying those that are more severe. Various scoring systems have been created in order to quickly assess the severity of injury and decide on the urgency and level of care required to keep that casualty alive. Where resources are limited, either on the battlefield or pressed urban mass-casualty situations, these scoring systems prevent wastage of resources on non-life-threatening injuries and, more controversially, non-survivable injuries (particularly head and brain injury). In these pressured environments, decisions made on the results of scoring systems can ultimately influence whether a casualty receives treatment or is managed expectantly even within hospitals. Some scoring systems are used as triage tools at point of injury, and others are used retrospectively (eg, Injury Severity Score, ISS). However, scoring 
Table 1 Fatality rate by year and corresponding admissions for neurorehabilitation

\begin{tabular}{lllllllllrrrr}
\hline Year & 2002 & 2003 & 2004 & 2005 & 2006 & 2007 & 2008 & 2009 & 2010 & 2011 & 2012 & 2013 \\
\hline $\begin{array}{l}\text { Total fatalities in Afghanistan } \\
\text { Total requiring neurorehabilitation }\end{array}$ & 3 & 0 & 1 & 1 & 39 & 42 & 51 & 108 & 103 & 46 & 44 & 9 \\
\hline
\end{tabular}

systems are based on the theoretical cumulative effect on body systems, and it is assumed that this accurately reflects nonsurvivability, morbidity and hospital stay, for instance. Indeed, doubt has already been raised in terms of the predictive ability of these scores, particularly in severe head injury. ${ }^{1} 2$ This study explores this association further by looking at the long-term outcomes for all operational traumatic brain injury admissions at a UK military neurorehabilitation unit and initial data at or near the time of injury. Further analysis is performed to evaluate whether initial ISSs reflect predicted outcomes, and indeed, if these scores are suitable for future planning in assessing whether a casualty offers sufficient potential for intensive rehabilitation.

\section{METHODS}

A retrospective database review was conducted by collating information from electronic and paper medical records for every UK military patient admitted as an inpatient to the Neurorehabilitation Unit at the Defence Medical Rehabilitation Centre (DMRC), Headley Court, due to injuries sustained during any operational tour from 2008 to 2012. This was conducted as part of a service evaluation, and approved by the local DMRC Ethics Committee for the purpose of service improvement. The primary archive database used to obtain information was the Joint Theatre Trauma Registry (JTTR), which is a Ministry of Defence-run collaboration that amalgamates all morbidity and mortality information on casualties sustained in operation theatres. Patients were initially identified through local records, and the raw unfiltered JTTR data were then formally requested. The dates for all inpatient admissions were identified, and each patient record within the JTTR was selected and analysed for coded data or free text recording of injury and trauma measures. Subsequent data were gathered from the neurorehabilitation database at DMRC (cross-referenced with JTTR) to look for outcome measures during individual admissions, final outcomes on discharge and those at review 4 months post discharge. Finally, all patients were followed to get up-to-date evidence of current employment and health status. Not all outcome measures were recorded electronically for each patient, and in these situations, paper records were consulted.

Data were collected for the following parameters: date of injury, sex, Service (Navy/Army/RAF), GCS, ISS, injury mechanism, total number of injuries, vocational outcome, living environment, roulement and survival. No patients were excluded from this study over the 4-year period. This dataset was subsequently transcribed manually to a spreadsheet that allowed comparison of raw data for each of the 12 selected data fields. Pictorial, tabular and graph representations were then used to explore the relationships between the various datasets. Statistical analyses on the raw and processed data were not performed due to the small overall dataset.

\section{RESULTS}

Overall, 34 casualties required inpatient neurorehabilitation from one tour of Iraq (Operation TELIC) and eight consecutive tours of Afghanistan (Operation HERRICK) covering 4 years,
5 months and 10 days between February 2008 and July 2012. This time period represents the heaviest combat activity for UK forces in the Afghanistan campaign (Table 1).

All of these patients were included within this retrospective analysis, and this cohort represents all those casualties with brain injuries, as a component of their overall injuries, extensive enough to require inpatient neurorehabilitation. All study participants were male; $22(65 \%)$ casualties were private soldiers or equivalent rank, 11 (32\%) were non-commissioned officers and one $(3 \%)$ was an officer. Overall, $94 \%$ of injuries were sustained through hostile action and $6 \%$ due to non-hostile action. UK forces employ a 6-month roulement where the current ground force is replaced by a fresh fighting force-the largest number of survivors of brain injury were sustained during the deployment roulement April 2009-October 2010 (Op HERRICK 10 and 11) (Figure 1). Numerically, the majority of UK Armed Forces are ground troops (ie, Army), and these personnel have greatest exposure to enemy activity-29 (88\%) casualties were Royal Army, three (9\%) were Royal Navy (all Royal Marines) and there was one (3\%) Royal Air Force member.

The most common injuries (44\%) were sustained through encounters with improvised explosive devices (IEDs), 41\% through gunshot wounds, 6\% from rocket-propelled grenades and remainder through mortars, mines and burns; $70.9 \%$ were penetrating wounds, and the remainder comprised blast and blunt injuries, with $17.6 \%$ showing a combined mechanism of injury. Head injury was the highest category of injury (according to Abbreviated Injury Score) in $76.5 \%$ (26) casualties. Although head injury was not considered the most serious injury in eight casualties, all had sustained brain injury of sufficient severity to qualify for inpatient neurorehabilitation (Table 2). There was an average of 11.7 injuries per person across this population-this represents the total injuries identified, from which, the three most severely injured body regions were used to calculate the ISS.

Initial GCS was recorded in $26(76 \%)$ cases at or soon after the point of injury, 11/26 (42\%) had a GCS score of 3 (severe

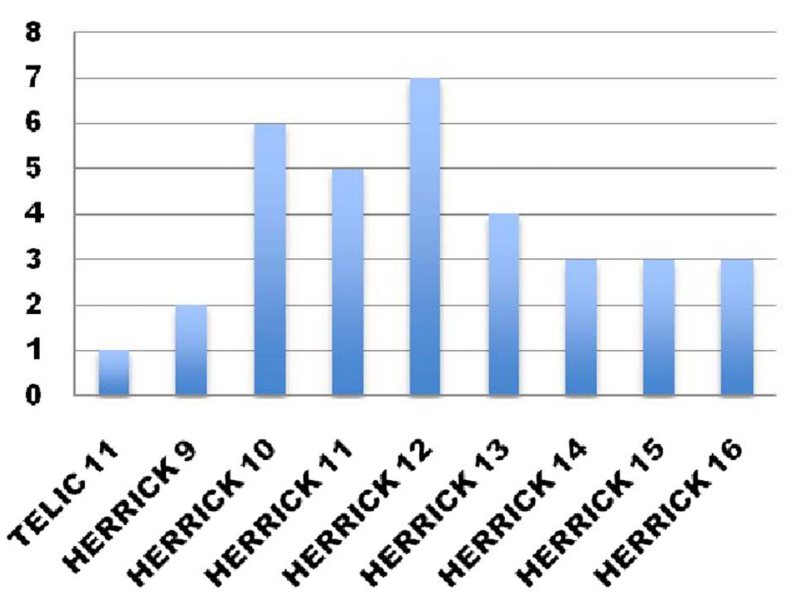

Figure 1 Number of casualties with brain injuries requiring neurorehabilitation according to deployment roulement 
Table 2 Injury breakdown

\begin{tabular}{|c|c|c|c|c|c|c|}
\hline Primary area of injury & Head & Thorax & Abdomen & Upper limb & Lower limb & Burns \\
\hline Proportion (n) of total casualties affected & $76.5 \%(26)$ & $5.9 \%(2)$ & $2.9 \%(1)$ & $2.9 \%(1)$ & $8.8 \%(3)$ & $2.9 \%(1)$ \\
\hline Injury type (top three injuries) & Penetrating & Combined & Blast & Blunt & & \\
\hline Proportion (n) of casualties affected & $70.9 \%(24)$ & $17.6 \%(6)$ & $5.9 \%(2)$ & $5.9 \%(2)$ & & \\
\hline Total number of injuries & $>10$ & $3-9$ & 2 & 1 & & \\
\hline Proportion and number of casualties affected & $26.5 \%(9)$ & $52.9 \%(18)$ & $8.8 \%(3)$ & $11.8 \%(4)$ & & \\
\hline
\end{tabular}

brain injury), one (3\%) had a score of 11 (moderate brain injury) and 14 (54\%) had a score of $>13$ (mild brain injury). ${ }^{3}$

All casualties had ISS recorded: 'Major trauma' is defined as an ISS of $\geq 15 ; 28 / 34(82 \%)$ had an ISS $>15$ and $16(47 \%)$ had an ISS of 75 at or soon after the point of injury, which is considered an 'unsurvivable' injury.

At 4 months after discharge following completion of inpatient neurorehabilitation at DMRC, $16(47 \%)$ casualties were fully independent, and 14 (41\%) were independent in their own homes and independent with personal care, but needed help with some things, such as planning journeys and managing household bills (Figure 2). Two (6\%) required help in one or more areas of personal care, and one patient (3\%) required fulltime help (ie, full-time carers); overall, 30/34 (88\%) were able to look after themselves in their own homes.

In total, $26(79 \%)$ casualties returned to either full-time or part-time work, and 11 (32\%) initial casualties returned to fulltime military duties (Figure 3); two (6\%) returned to vocational education, and were deemed capable of returning to work based on vocational assessment, and six (18\%) went on to have vocational rehabilitation in the community, and were capable of returning to work after completion. Only two patients (6\%) were not capable of returning to work.

Two casualties $(12.5 \%)$ with an ISS of 75 returned to fulltime military duties, and seven of those returning to full-time military duties (64\%) had an ISS of $>15$; four casualties who returned to military duties had ISS values $<4$. In summary, $7 / 11$ casualties who returned to military duties had major trauma, and two were deemed 'unsurvivable'.

Fifteen (93\%) of the 16 casualties who had an ISS of 75 , and $10 / 11(91 \%)$ with a GCS of 3 were capable of returning, or have returned, to either full-time or part-time employment. Looking at the most severely affected according to both scoring systems, there

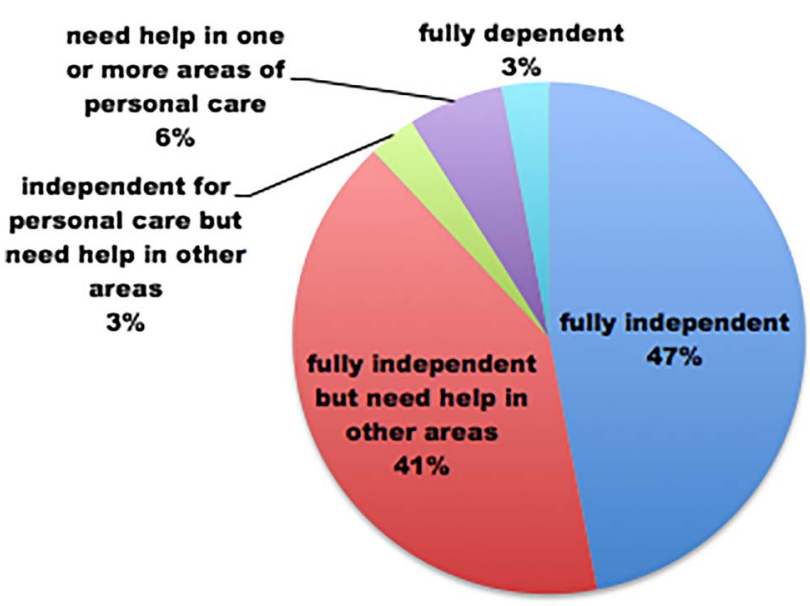

Figure 2 Ability to live independently and self-care after rehabilitation. were seven casualties with both GCS of 3 and ISS of 75-all survived and returned to a level of independence in their own homes, but required help with some activities, such as paying bills.

\section{DISCUSSION}

The analysis of this cohort of unselected military patients with brain injury shows that poor ISS and GCS scores do not accurately predict or reflect eventual outcome. ISS should not be used to predict long-term prognosis, quality of life or employability as brain injury was shown to be recoverable.

The recent UK operational medical structure for military personnel at the point of injury in battle zones is extensive, well tested and based on many years of experience in treating injured personnel in different wars in differing locations. Battlefield trauma management encompasses rescue and recovery from rough terrain in inhospitable conditions to a modern medical facility with medical experts and advanced equipment designed to maximise survival. The confidence this lends to troops on the ground contributes to the moral component of fighting power. This knowledge, namely that they will survive if injured, creates a forward-facing force that will engage in battle and win conflicts. Survival, however, is not the endpoint of medical management, but the start, and the ambition has now moved beyond simply maintaining life to returning injured patients to full activity and fulfilling work.

Conducting an analysis on war casualties undergoing neurorehabilitation over this 4-year period presented certain challenges and identified potential limitations to this study. The retrospective nature of this research meant complete reliance on historical records, and peculiar to this study, the military aspect of using sensitive information concerning patients who were still officially on active duty. Information gathering is highlighted as another potential limitation where statistics have been collected in different

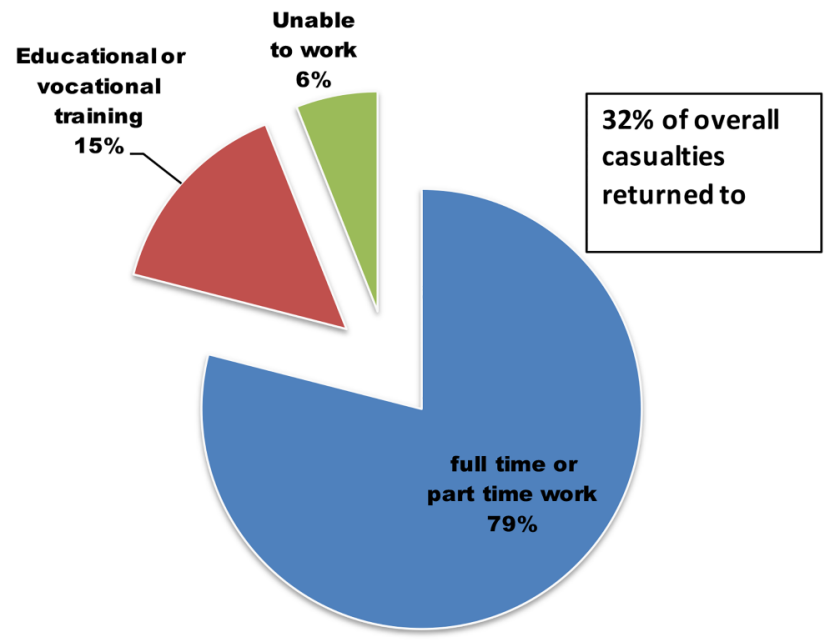

Figure 3 Vocational outcome 4 months after discharge from rehabilitation. 
conditions in a variety of environments (eg, point of wounding, field hospitals, UK tertiary centres) by different observers. The patient cohort represents survivors, and those personnel, who were fatally wounded, were not included. The evolving nature of neurorehabilitation over this 4 -year period has meant that treatment and outcome assessment has changed as the needs and understanding of our military-wounded patients have changed. During the timelines of this study, a standardised approach to collecting employment outcomes had not been adopted, whereas internationally recognised tools, such as 'MPAI-4' (Mayo-Portland Adaptability Inventory-an evaluation tool for brain injury) is now used for all such patients at DMRC, Headley Court. Additionally, a larger sample size would lend more weight to the findings, as there were only 34 participants within this study. Previous research in this area has demonstrated that penetrating brain injury in military patients tends to show better survival than predicted. ${ }^{4}$ However, this was based on survival to the point of discharge from the treating hospital. The results of this study build on these findings by following patients beyond discharge into community independence and employment.

The difficulties faced by military clinicians in warfare, and by civilian clinicians during mass casualty situations, include the understandable concern that may come to mind questioning whether eventual quality of life after survival will be severely impaired. Where resources are limited, for example, in emergent mass casualty situations, these concerns may influence the care given, particularly where previous opinion may have suggested that high ISSs are thought to predict eventual outcome. However, the results in this 4-year analysis consistently demonstrate that ISS in the severe range does not predict patient outcome. All the 'unsurvivable' survived and completed neurorehabilitation, and the vast majority returned to employment with a significant number returning to military duties.

An analysis of the mechanism of injury shows that IED and gunshot wounds account for the majority of injuries (85\%), which is an accurate reflection of warfare, in that both these weapon systems are user-operated and targeted against individuals, rather than opportunistic (eg, land mines). Indeed, the aim of many land mines is not to kill, but simply disable, thereby forcing personnel to slow down and stop while casualties are evacuated. In contrast, IED strikes are often activated in direct line of sight and placed above ground in road signs/trees, where maximal damage can be inflicted. With this in mind, an IED strike or gunshot wound should alert the clinician to the possibility of serious brain injury even if information has been communicated in advance about the status of the casualty. This is further supported by the finding that most casualties $(79.4 \%)$ in this study had three or more injuries from the same incident, with an average of 11.7 injuries per casualty. Further analysis of this mechanism of injury shows that $70.9 \%$ of patients, requiring inpatient brain injury rehabilitation, had suffered penetrating wounds to the head. High-energy penetrating brain injury is expected to cause severe neurological dysfunction, but in onequarter of cases, the top primary and secondary injuries did not involve penetrating head wounds. The injury profile in these cases represented lower limb amputation (33\%), lung injury (22\%), upper limb amputation (11\%), liver laceration (11\%), extensive tongue laceration (11\%) and second-degree burns $(11 \%)$. However, these casualties still suffered moderate or severe brain injury, despite other regions being the main concern. This again supports the finding that any IED/gunshot wound should be an indication of possible neurological injury as both involve high-energy projectiles and fragments designed to penetrate tissue. Indeed, neurological injury should be looked for in all significant injuries irrespective of body region affected, as $8.8 \%$ of those admitted for intensive inpatient brain injury rehabilitation had actually sustained a lower limb injury as their most severe injury. It is, therefore, important to assess neurological injury in all patients with multiple injuries irrespective of whether head/neck injury was initially apparent.

Initial GCS was unfortunately not recorded in all casualties, and this could be as much a fault of poor documentation as lack of measurement. While GCS has many flaws in terms of predictive ability, extreme values are useful (ie, GCS 3 corresponds to the most severe injury). Out of the 11 patients who had an initial GCS 3, 10 have gone back to employment or higher education. GCS corresponded well with overall ISS in that all cases of GCS 3 had an ISS >15 (ie, major trauma). This could reflect previous findings that low GCS may not be primarily due to brain injury, but a reflection of the other injuries sustained. ${ }^{5}$ Both scales reflect different physiological parameters, and close association would only be expected at extremes in such small patient samples. ISS is certainly the preferred retrospective tool for better assessment of overall injury compared with GCS. ${ }^{6}$ Indeed, where GCS primarily describes neurological deficit at a point in time, ISS is based on providing a single figure that retrospectively summarises injury to various anatomical regions in patients with multiple injuries. GCS can be performed at the point of injury, and is used in triage (although during its inception, it was intended as a postresuscitation score), whereas ISS relies on greater knowledge of injury, and not every injury is apparent until a more thorough assessment has been performed, precluding its usefulness as a triage tool.

Even with these recognised limitations, ISS has been revised and updated, and it is still accepted as the only score that correlates with mortality, morbidity and duration of inpatient stay. However, in this study population, all of those casualties deemed 'unsurvivable' (ISS=75) left hospital and rehabilitation successfully, and in terms of long-term morbidity, 91\% were fully self-caring after rehabilitation. Indeed, on follow-up, 97\% of those casualties deemed 'unsurvivable' were able to return to work (full time or part time) or education. Other factors could contribute to this high recovery rate. It is known that nutritional status ${ }^{7}$ and premorbid educational level are important in functional recovery, ${ }^{8}$ and there are a number of other factors that contribute significantly to short-term and long-term positive outcome, particularly the improvements in acute casualty care driven by recent conflicts. However, initial treatment cannot fully explain the positive outcomes seen in this analysis. Improved trauma management has led to greater survivability only in moderate-severe injuries, and in-hospital mortality rates for both 'least severe' and 'most severe' injuries remain unchanged. ${ }^{9}$ A major factor in the positive results seen over this 4-year period is the delivery of inpatient consultant-led multidisciplinary neurorehabilitation where patients have access to rehabilitation focused on achieving independence and return to meaningful employment. Patients' rehabilitation needs are assessed over a 3-week period, allowing the generation of an individualised rehabilitation programme tailored to their needs and aspirations. The multidisciplinary team deliver this programme through goal-based activities that typically comprise 35 intensive sessions per week. Thus, goal-driven rehabilitation tailored to individual patient needs can be delivered rather than generic therapy, and investment in this approach is reflected in the results-patients with severe traumatic brain injury returning to independent living and meaningful employment.

The differences in actual outcome over expected (or predicted) outcome may be due to the dataset used to create and 
stratify these scoring systems. The calibration data and any updates (at least more than 15 years ago) originally used when these systems were created do not reflect the improved knowledge of trauma care and rehabilitation. Indeed, this potential failing has already been identified in a 20 -year study ${ }^{10}$ of trauma patients and their outcomes where scoring predictions did not correlate well with actual outcomes.

\section{CONCLUSIONS}

Brain injury is often regarded as having a much greater negatively prognostic effect than other injuries, particularly in traumatic situations. ISS are retrospective instruments, and are not used to prognosticate casualty management; however, ISS scores deemed 'unsurvivable' imply that the long-term functional outcome and quality of life will be poor. Overall, the results suggest that these injury severity scoring systems should not be used either for consideration of 'expectant' management or as a definite predictor of long-term permanent neurological functional deficit, as they are poor predictors of long-term survivability, quality of life and employability. Frontline clinicians should be encouraged to actively intervene and treat patients with severe traumatic brain injury with the knowledge that full recovery is possible and probable, and independence and return to employment are not just achievable, but very likely.

Correction notice This article has been corrected since first appearing in print. The correspondence details have been changed.
Contributors Main document by SB. Overall review by EMG and JE.

Competing interests None declared.

Provenance and peer review Not commissioned; externally peer reviewed.

\section{REFERENCES}

1 de Jongh $M$, Verhofstad M, Leenen L. Accuracy of different survival prediction models in a trauma population. Br J Surg 2010;97:1805-13.

2 Toschlog EA, MacElligot J, Sagraves SG, et al. The relationship of Injury Severity Score and Glasgow Coma Score to rehabilitative potential in patients suffering traumatic brain injury. Am Surg 2003;69:491-7.

3 Teasdale G, Jennett B. Assessment of coma and impaired consciousness. Lancet 1974;304:81-4.

4 Smith JE, Kehoe A, Harrisson SE, et al. Outcome of penetrating intracranial injuries in a military setting. Injury 2014;45:874-8.

5 Grote S, Böcker W, Mutschler W. Diagnostic value of the Glasgow Coma Scale for traumatic brain injury in 18,002 patients with severe multiple injuries. J Neurotrauma 2011;28:527-34.

6 Foreman B, Caesar R, Parks J, et al. Usefulness of the abbreviated injury score and the injury severity score in comparison to the Glasgow Coma Scale in predicting outcome after traumatic brain injury. J Trauma 2007;62:946-50.

7 Hoffmann M, Rueger J. Nutritional status influences trauma outcome. Der Unfallchirurg 2011;114:968-72.

8 Hotslag $H$, van Beeck E, Lindemann $E$, et al. Determinants of long-term functional consequences after major trauma. J Trauma 2007;62:919-27.

9 Glance L, Osler T, Mukamel D, et al. Outcomes of adult trauma patients admitted to trauma centers in Pennsylvania, 2000-2009. Arch Surg 2012; 147:732-7.

10 Roger $\mathrm{F}$, Osler T, Kirasne M, et al. Has TRISS become an anachronism? A comparison of mortality between the National Trauma Database and Major Outcome Study Database. J Trauma Acute Care Surg 2012;73:326-31. 